\title{
ESTUDIO DE OLAS DE CALOR EN CONDICIONES DE CLIMA FUTURO (2071-2100) A PARTIR DE UN CONJUNTO DE MODELOS REGIONALES DE CLIMA DE LA BASE DE DATOS EURO-CORDEX
}

\author{
María Ofelia Molina ${ }^{(1)}$, Enrique Sánchez ${ }^{(1)}$, Claudia Gutiérrez $^{(1)}$. \\ (1) Facultad de Ciencias Ambientales y Bioquímica, Universidad de Castilla-La Mancha \\ (UCLM) - Av. Carlos III s/n 45071 Toledo (Spain). \\ MOfelia.Molina1@alu.uclm.es
}

\section{Resumen}

Las olas de calor son uno de los eventos climáticos extremos más preocupantes debido a la vulnerabilidad de nuestra sociedad a sus efectos adversos y el probable incremento de su frecuencia y duración a lo largo del siglo XXI. La duración y la intensidad de la ola de calor son los dos aspectos fundamentales a tener en cuenta en su definición.

En este trabajo seleccionamos el índice HWMI (Heat Wave Magnitude Index, Russo et al., 2014) para su análisis. Este índice estudia la relación entre la intensidad, entendida como la anomalía de temperatura de la ola de calor con respecto a una temperatura de referencia, y su longitud. A partir de esto se obtiene un índica adimensional que caracteriza la ola de calor.

La duración y la intensidad de las olas de calor se analizan sobre Europa usando simulaciones de un conjunto de modelos regionales de clima incluidos dentro de la base de datos EURO-CORDEX (http://www.euro-cordex.net/)(Jacob et al., 2014). Vautard et al. (2013) es el trabajo de referencia donde se muestra la capacidad de los modelos para representar los eventos extremos en condiciones de clima actual, como primer paso necesario para poder tener fiabilidad sobre las proyecciones futuras que simulen.

En un primer paso del análisis de la base de datos de simulaciones de EURO-CORDEX, se propone la comparación de pares de simulaciones en los que hay sólo cambios bien en la resolución $\left(0,44^{\circ}\right.$ frente a $\left.0,11^{\circ}\right)$, bien en el modelo regional, bien en el modelo global que los fuerza o bien en el escenario de emisiones de gases de efecto invernadero (RCP4.5 frente a RCP8.5). Se analiza la evolución en las características de las olas de calor y se proyecta que aumenten su intensidad y duración a finales del siglo XXI. En cuanto a la sensibilidad a los diferentes factores, se indica que el efecto de usar un GCM u otro es importante, no siendo así el efecto del RCM, que una mayor resolución permite un análisis más exhaustivo de los resultados y que ante un escenario de más emisiones de gases, las olas de calor serás más duraderas y de mayor magnitud. No obstante, se trata de una comparación todavía limitada a pares de simulaciones, a falta de una comparación más exhaustiva y completa del efecto de cada uno de estos elementos.

\section{Introducción}

Las olas de calor son uno de los eventos climáticos extremos más preocupantes debido a la vulnerabilidad de nuestra sociedad a sus efectos adversos y el probable in- 
cremento de su frecuencia y duración a lo largo del siglo XXI (Frich, P. et al., 2002, IPCC, 2013, Sánchez et al., 2017).

Una ola de calor es un periodo de días consecutivos en que las temperaturas son elevadas. Existen diferentes definiciones de olas de calor (Perkins, 2015), pero una descripción completa debería incluir la duración y la intensidad global de la misma como aspectos fundamentales.

Algunas de las condiciones sinópticas que deben reunirse para que una ola de calor se produzca, son las de un sistema de altas presiones que permanece en un lugar durante un periodo prolongado de tiempo que, al advectar aire cálido y seco, hace que las altas temperaturas se mantengan durante más tiempo (Perkins, 2015). Esta situación se ve favorecida en regiones con suelos secos o humedad baja que amplifican la probabilidad de que las temperaturas sean extremas (Sillmann et al., 2017).

La ETCCDI (Expert Team on Climate Change Detection and Indices, http://etccdi. pacificclimate.org/) ha desarrollado varios índices climáticos para medir las temperaturas extremas (Zhang et al., 2011). El WSDI (Warm Spell Duration Index) o el TX90p, calculados a partir de umbrales basados en percentiles, son los más comúnmente usados para estudiar la frecuencia de ocurrencia de las olas de calor. Este tipo de índices basados en percentiles, muestran la variación de temperatura con respecto a un umbral, que es el percentil 90 de un periodo de referencia (1961-1990). El WSDI es un buen índice para comparar la duración en días de las olas de calor espacialmente.

La magnitud cuantificada con el HWMI viene de la relación entre la intensidad, entendida como la anomalía de temperatura de la ola de calor con respecto a una temperatura de referencia, y la duración de esa ola de calor. Es un índice adimensional que ofrece los dos aspectos clave en el estudio de las olas de calor: la duración y la intensidad.

En Vautard et al. (2013) se estudió la capacidad de los modelos regionales de EUROCORDEX (Jacob et al., 2014) para representar las olas de calor en clima presente (1989-2008) mediante una Distribución de Pareto Generalizada (GPD). El trabajo aquí presentado estudia también los modelos regionales de EURO-CORDEX y cómo simulan las olas de calor bajo condiciones de clima futuro (2071-2100) bajo dos escenarios de emisiones, $\mathrm{RCP} 4.5$ en el que la concentración de $\mathrm{CO}_{2}$ alcanza 538 ppm y RCP8.5, en el que las emisiones alcanzan 936 ppm en el año 2100 (Moss et al., 2010).

\section{Objetivos}

El objeto principal de este trabajo es estudiar la duración e intensidad de las olas de calor en condiciones de clima futuro (2071-2100) sobre Europa, obtenida con los modelos regionales de EURO-CORDEX. Junto a este objetivo, se plantea el análisis de la evolución de las olas de calor con respecto al clima actual (1971-2000) así como la evaluación de simulaciones con distintos escenarios de emisiones (RCP4.5 vs RCP8.5), y la comparación de resultados entre pares de simulaciones regionales en los que hay sólo cambios bien en la resolución $\left(0,44^{\circ}\right.$ frente a $\left.0,11^{\circ}\right)$, bien en el modelo regional (RCM) o bien en el modelo global forzante (GCM). 


\section{Metodología}

Este estudio se centra en el análisis de las olas de calor a partir de las salidas de temperatura máxima diaria de algunas de las simulaciones regionales de clima EURO-CORDEX, utilizando diferentes modelos y resoluciones. Las simulaciones de EURO-CORDEX usan como condiciones de contorno para forzar los modelos regionales las simulaciones climáticas globales de CMIP5 hasta el año 2100. Se basan en escenarios de emisión de gases de efecto invernadero (Representative Concentration Pathways, RCP) correspondientes a la estabilización del forzamiento radiativo después del siglo XXI a 4,5 W/ $/ \mathrm{m}^{2}$ (RCP4.5), forzamiento radiativo ascendente que supera $8,5 \mathrm{~W} / \mathrm{m}^{2}$ a fines del siglo XXI (RCP8.5) y un forzamiento radiativo máximo en el siglo XXI de $3,0 \mathrm{~W} / \mathrm{m}^{2}$ y una disminución después (RCP2.6, también conocido como RCP3-PD) (Moss et al., 2008 y 2010; Nakicenovic et al., 2000). Para este estudio, se han seleccionado las simulaciones correspondientes a los escenarios de emisiones RCP4.5 y RCP8.5.

Para poder comparar los valores de HWMI entre modelos, se han seleccionado dos Modelos Regionales de Clima (RCMs), RCA y RACMO. Para poder estudiar el efecto que la resolución ejerce sobre los modelos climáticos para simular las olas de calor, se ha seleccionado el RCM RCA forzado por el GCM EC-EARTH y se ha calculado el HWMI con dos resoluciones diferentes para el RCM: 0,44 (EUR-44, $\sim 50 \mathrm{~km}$ ) y $0,11^{\circ}$ (EUR-11, $\sim 12.5 \mathrm{~km}$ ). Por último, para ver el efecto que produce cambiar los forzamientos en los contornos del RCM, se han seleccionado dos Modelos Globales de Clima (GCMs), CCC y EC-EARTH.

La elección de un buen índice para calcular las olas de calor depende del objetivo de estudio, en este caso el índice seleccionado es el HWMI (Russo et al. 2014). El HWMI analiza las temperaturas máximas diarias comparadas con el periodo de referencia 19812010, y su duración. De este modo, agrupa las olas de calor en siete categorías, desde 1 (normal) hasta 32 (ultra extremo). Los cálculos han sido realizados con el paquete de software libre R "extRemes" (https://cran.r-project.org/web/packages/extRemes/extRemes.pdf). Una vez obtenido el HWMI de toda la muestra de datos, se realiza el promedio a 30 años del HWMI y se representa graficamente para poder comparar los resultados.

\section{Resultados y discusión}

En este apartado se muestran dos de los resultados que se obtienen al calcular el HWMI con el paquete de R "extRemes", el propio índice HWMI y la duración de la ola de calor más larga durante el periodo analizado, ambos promediados a 30 años.

Bajo el escenario de emisiones más severo (RCP8.5), la magnitud de la ola de calor es mayor que bajo el escenario RCP4.5 independientemente del modelo regional o global. Esto es, en condiciones de un incremento mayor de gases de efecto invernadero, las olas de calor tendrán una mayor duración e intensidad.

Comparando entre escenarios de emisiones, el RCP4.5 muestra sobre Europa valores del HWMI (Figura 1) entre 1 y 2, aunque RCA-CCC muestra valores de 5 en zonas del Mediterráneo. Con RCP8.5, el HWMI asciende a valores de 2 y 3 en la mayor parte del dominio, alcanzando valores ente 10 y 15 en el Mediterráneo. Estos resultados parecen consistentes comparándolos con los resultados obtenidos por Russo et al. 2014, donde 
se muestran las series temporales promedio del HWMI para el periodo 1980-2100 sobre Europa Central con el conjunto de modelos CMIP5 y los escenarios RCP2.6, RCP4.5 y RCP8.5. La duración de las olas de calor más severas en el futuro (Figura 2), bajo el escenario $\mathrm{RCP} 4.5$, se prevé que esté entre 4 y 10 días según el modelo, y bajo el escenario RCP8.5 entre 8 y 10 días, superando los 20 días en los casos más extremos. El escenario más severo es el mostrado por RCA bajo el forzamiento de CCC y bajo el escenario de emisiones RCP8.5, donde la ola de calor supera los 10 días de duración en todo el territorio estudiado.
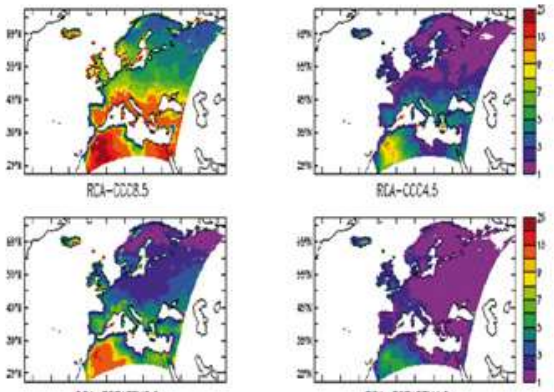

BCH-ECEATh

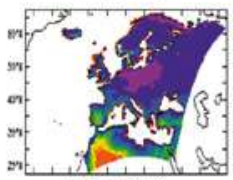

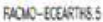

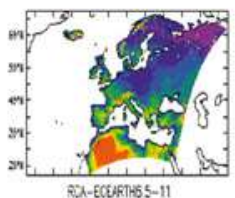

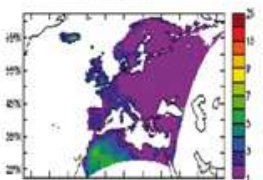

RCA-EEEATHAS

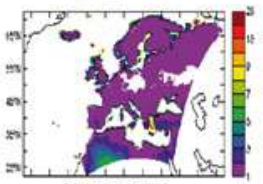

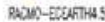

AC-EXERTH.5.11

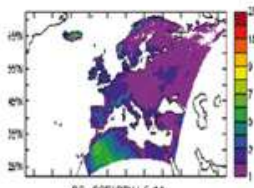

Figura 2. Días promedio de la duración de la ola de calor más larga durante el periodo 2071-2100 según los cálculos del script R para obtener HWMI, comparando los GCMs CCC vs EC-EARTH, los RCMs RCA vs RACMO y

los escenarios RCP4.5 vs RCP8.5. En la columna de la izquierda se muestran las figuras correspondientes al RCP8.5 y, a la derecha, el escenario RCP4.5. La primera fila corresponde al escenario mostrado por RCA bajo el forzamiento de CCC, la segunda fila corresponde al escenario mostrado por RCA bajo el forzamiento de EC-EARTH y la tercera fila corresponde al escenario de RACMO forzado por EC-EARTH.

Figura 1. Promedio del HWMI para el periodo 2071-2100, comparando los GCMs CCC vs ECEARTH, los RCMs RCA vs RACMO, los escenarios RCP4.5 vs RCP8.5 y la resolución $0,11^{\circ}$ vs $0,44^{\circ}$. En la columna de la izquierda se muestran las figuras correspondientes al RCP8.5 y, a la derecha, el escenario RCP4.5. La primera fila corresponde al escenario mostrado por RCA bajo el forzamiento de CCC, la segunda fila corresponde al escenario mostrado por RCA bajo el forzamiento de ECEARTH, la tercera fila corresponde al escenario de RACMO forzado por EC-EARTH y la cuarta fila al es igual que la segunda, pero con una resolución de $0,11^{\circ}$, el resto tienen una resolución de $0,44^{\circ}$.
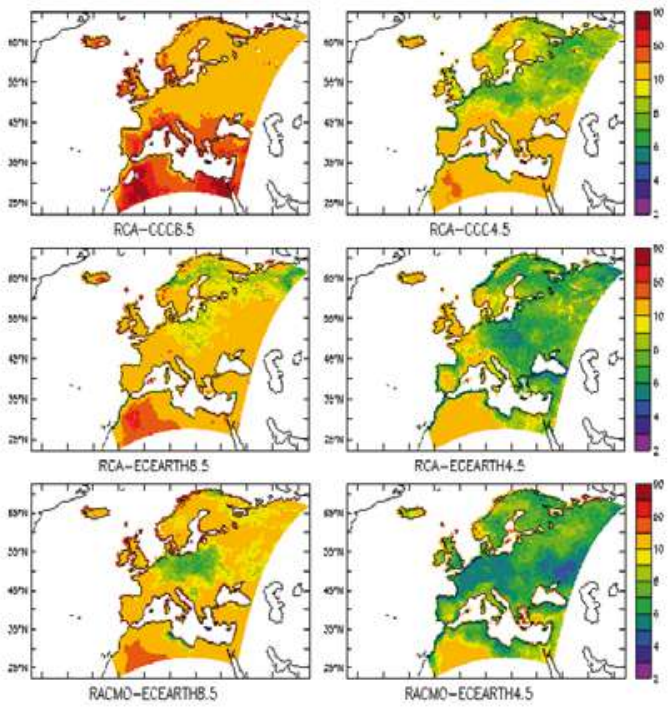
En cuanto a la comparación de los resultados entre GCMs, tanto el HWMI como la duración de la ola de calor más severa, son sensiblemente mayores en el escenario que muestra el RCM RCA bajo el forzamiento del GCM CCC que bajo el forzamiento del EC-EARTH en los dos escenarios de emisiones, por lo que el efecto de usar un GCM u otro parece importante y merece la pena ser analizado en profundidad.

Sin embargo, aparentemente, el efecto del RCM (comparando RCA con RACMO) parece ser menor. Entre RCMs, forzados ambos por el mismo GCM, el RCA muestra valores de duración de la ola de calor ligeramente más altos que RACMO en los dos escenarios de emisiones.

El aumento de la resolución de $0.44^{\circ}$ a $0.11^{\circ}$ permite visualizar detalles espaciales en zonas de orografía compleja o costera, permitiendo mostrar estructuras más detalladas en muchas partes del dominio.

El trabajo, que está en marcha, pretende profundizar en el estudio de más modelos regionales disponibles que los aquí mostrados, diferentes modelos globales forzantes, otras magnitudes para analizar olas de calor, el efecto de la resolución y la comparación de los cambios respecto a condiciones de clima actual, para intentar obtener una imagen lo más completa posible de las olas de calor para clima futuro sobre Europa a partir de las simulaciones regionales de EURO-CORDEX.

\section{Referencias}

- Frich, P., Alexander, L. V., Della-Marta, P., Gleason, B., Haylock, M., Klein Tank, A. M. G. and Peterson, T., 2002: Observed coherent changes in climatic extremes during the second half of the twentieth century, Climate Research, 19, 193-212.

- Jacob, D., Petersen, J., Eggert, B., Alias, A., Christensen, O.B., Bouwer, L.M., Braun, A., Colette, A., Déqué, M., Georgievski, G., Georgopoulou, E., Gobiet, A., Menut, L., Nikulin, G., Haensler, A., Hempelmann, N., Jones, C., Keuler, K., Kovats, S., Kröner, N., Kotlarski, S., Kriegsmann, A., Martin, E., Meijgaard, E., Moseley, C., Pfeifer, S., Preuschmann, S., Radermacher, C., Radtke, K., Rechid, D., Rounsevell, M., Samuelsson, P., Somot, S., Soussana, J., Teichmann, C., Valentini, R., Vautard, R., Weber, B. and Yiou, P., 2014: EURO-CORDEX: new high-resolution climate change projections for European impact research, Regional Environmental Change, 14, no. 2, 563-578.

- Moss, R., Babiker, M., Brinkman, S., Calvo, E., Carter, T., Edmonds, J., Elgizouli, I., Emori, S., Erda, L., Hibbard, K.A., Jones, R., Kainuma, M., Kelleher, J., Lamarque, J.F., Manning, M., Matthews, B., Meehl, J., Meyer, L., Mitchell, J., Nakicenovic, N., O’Neill, B., Pichs, R., Riahi, K., Rose, S., Runci, P., Stouffer, R., van Vuuren, D., Weyant, J., Wilbanks, T., van Ypersele, J.P. and Zurek, M., 2008: Towards New Scenarios for Analysis of Emissions, Climate Change, Impacts, and Response Strategies. Intergovernmental Panel on Climate Change, Geneva, 132.

- Moss, R.H., Edmonds, J.A., Hibbard, K.A., Manning, M.R., Rose, S.K., van Vuuren, D.P., Carter, T.R., Emori, S., Kainuma, M., Kram, T., Meehl, G.A., Mitchell, J.F.B., Nakicenovic, N., Riahi, K., Smith, S.J., Stouffer, R.J., Thomson, A.M., Weyant, J.P., Wilbanks, T.J., 2010: The next generation of scenarios for climate change research and assessment. Nature, 463(7282), 747-756.

- Nakicenovic, N., Alcamo, J., Davis, G., et al., 2000: Special Report on Emissions Scenarios, Intergovernmental Panel on Climate Change, Cambridge University Press, Cambridge, United Kingdom and New York, 599.

- Perkins, S. E., 2015: A review on the scientific understanding of heatwaves-Their measurement, driving mechanisms, and changes at the global scale, Atmospheric Research, 164-165, 242-267. 
- Russo, S., A. Dosio, R. G. Graversen, J. Sillmann, H. Carrao, M. B. Dunbar, A. Singleton, P. Montagna, P. Barbola, and J. V. Vogt, 2014: Magnitude of extreme heat waves in present climate and their projection in a warming world, J. Geophys. Res. Atmos., 119, 12,500-12,512.

- Sánchez , E., Rodríguez-Fonseca, B., Gutierrez, M., Jordá, G., Salat, J. and Valero-Garcés, B. L., 2017: Progress in Detection and Projection of Climate Change in Spain since the 2010 CLIVARSpain regional climate change assessment report. Special Issue on climate over the Iberian Peninsula: an overview of CLIVAR-Spain coordinated science, CLIVAR Exchanges, 73, 1-4.

- Sillmann, J., Thorarinsdottir, T., Keenlyside, N., Schaller, N., Alexander, L V., Hegerl, G., Seneviratne, S I., Vautard, R., Zhang, X., Zwiers, F W., 2017: Understanding, modeling and predicting weather and climate extremes: Challenges and opportunities, Weather and Climate Extremes, 18, 65-74.

- Stocker, D., Qin, G.K., Plattner, M., Tignor, SK., Allen, J., Boshchung, A., Nauels,Y., Xia, V, Bex, P.M., Midgley (Eds.), IPCC 2013 Climate Change, 2013: The Phisical Science Basis. Contribution onf Working Group I to the Fifth Assesment Report of the Intergovernmental Panel on Climate Change, Cambridge University Press, 1535.

- Vautard, R., Gobiet, A., Jacob, D., Belda, M., Colette, A., Déqué, M., Fernández, J., M García-Díez, M., Goergen, K., Güttler, I., Halenka, T., Karacostas, T., Katragkou, E., Keuler, K., Kotlarski, S., Mayer, S., van Meijgaard, E., Nikulin, G., Patarčić, M., Scinocca, J., Sobolowski, S., Suklitsch, M., Teichmann, C., Warrach-Sagi, K., Wulfmeyer, V. and Yiou, P., 2013: The simulation of European heat waves from an ensemble of regional climate models within the EURO-CORDEX Project, Climate Dynamics, 41, 2555-2575.

- Zhang, X., Alexander, L., Hegerl, G. C., Jones, P., Tank, A. K., Peterson, T. C., Trewin, B. and Zwiers, F. W., 2011: Indices for monitoring changes in extremes based on daily temperature and precipitation data, WIREs Clim Change, 2, 851-870. 